\title{
High expression of fructose-bisphosphate aldolase A induces progression of renal cell carcinoma
}

\author{
ZHENGKAI HUANG $^{1 *}$, YIBO HUA $^{1 *}$, YE TIAN $^{1 *}$, CHAO QIN $^{1}$, JIAN QIAN ${ }^{1}$, MEILING BAO $^{2}$, YIYANG LIU ${ }^{1}$, \\ SHANGQIAN WANG ${ }^{1}$, QIANG CAO ${ }^{1}$, XIAOBING JU ${ }^{1}$, ZENGJUN WANG ${ }^{1}$ and MIN GU $^{1}$ \\ Departments of ${ }^{1}$ Urology and ${ }^{2}$ Pathology, The First Affiliated Hospital of Nanjing Medical University, \\ Nanjing, Jiangsu 210029, P.R. China
}

Received September 11, 2017; Accepted April 13, 2018

DOI: $10.3892 /$ or.2018.6378

\begin{abstract}
Aldolase A (fructose-bisphosphate aldolase A, ALDOA) is a glycolytic enzyme that catalyzes reversible conversion of fructose-1,6-bisphosphate to glyceraldehyde 3 -phosphate and dihydroxyacetone phosphate. ALDOA has been revealed to be related with many carcinomas, but its expression and function in renal cell carcinoma (RCC) remain unknown. This study aimed to detect expression of ALDOA in human RCC tissue samples and to explore its function in RCC cell lines. Reverse transcription-polymerase chain reaction was used to quantify ALDOA in human RCC samples. A total of 139 RCC tissue samples obtained after surgery were analyzed in tissue microarray for ALDOA immunohistochemistry-based protein expression. Assays for cell cycle, viability, migration, and invasion were performed to assess phenotypic changes in RCC cells after ALDOA knockdown by small interfering RNA-mediated gene silencing approach and ALDOA upregulation by overexpression plasmids. Western blot analysis was used to identify alterations in markers for epithelial-mesenchymal transition (EMT), which affects metastasis and the $\mathrm{Wnt} / \beta$-catenin signaling pathway that influences RCC cell growth. ALDOA was upregulated in RCC samples and RCC cell lines $(\mathrm{P}<0.01)$. Expression of ALDOA was significantly associated with metastasis $(\mathrm{P}=0.020)$ and survival $(\mathrm{P}=0.0341)$. Downregulation of ALDOA suppressed proliferation $(\mathrm{P}<0.05)$ by triggering $\mathrm{G} 0 / \mathrm{G} 1$ cell cycle arrest $(\mathrm{P}<0.05)$ and also inhibited migration $(\mathrm{P}<0.05)$ and invasion $(\mathrm{P}<0.01)$. Upregulation of ALDOA promoted proliferation $(\mathrm{P}<0.05)$ and enhanced migration $(\mathrm{P}<0.001)$ and invasion $(\mathrm{P}<0.001)$. Low expression of ALDOA could reverse EMT
\end{abstract}

Correspondence to: Dr Min Gu or Dr Zengjun Wang, Department of Urology, The First Affiliated Hospital of Nanjing Medical University, 300 Guangzhou Road, Nanjing, Jiangsu 210029, P.R. China

E-mail: lancetgu@aliyun.com

E-mail: zengjunwang@njmu.edu.cn

*Contributed equally

Key words: renal cell carcinoma, ALDOA, EMT, Wnt and inactivate the Wnt/ $\beta$-catenin signaling pathway. Our data revealed that ALDOA functions as a tumor promoter, plays a prominent role in proliferation, migration, and invasion of RCC cells with high expression, and may promote EMT and activate the Wnt/ $\beta$-catenin signaling pathway.

\section{Introduction}

Renal cell carcinoma (RCC) is commonly diagnosed in urological malignant tumors. In 2016, the American Cancer Society estimated that the number of new kidney cancer cases would reach almost 62,000 in the United States, accounting for 1 in 20 new diagnoses in men and 3\% for women (1). An estimated 14,000 Americans will succumb to kidney cancer this year. In China, RCC is also one of the common diseases in urology. Projected age-standardized incidence rate (per 100,000) of kidney cancer reaches 2.4 in males and 1.0 in females (2). Although scientists have not identified exact causes of RCC occurrence, accumulating evidence suggests that obesity, hypertension, smoking, alcohol, occupational exposure to trichloroethylene, and genetic factors are risk factors for this disease (3).

$A L D O A$, also named $A L D A, G S D 12$ and $H E L-S-87 p$, encodes the protein aldolase A (or fructose-bisphosphate aldolase), which is a glycolytic enzyme that catalyzes reversible conversion of fructose-1,6-bisphosphate to glyceraldehyde 3-phosphate and dihydroxyacetone phosphate. Three different genes encode three aldolase isozymes (A, B and C). Aldolase A is the major aldolase in early embryos and adult muscles and is expressed lowly in adult liver, kidneys, and intestines (4). Aldolase A deficiency is associated with hemolytic anemia and severe rhabdomyolysis (5-7). High expression of ALDOA is related to lung squamous cell carcinoma (8), highly metastatic pancreatic cancer (9) and colorectal cancer (10). Researchers discovered that RCC patients feature elevated serum aldolase A compared with those with other urological tumors and benign urological diseases (11). A recent study identified ALDOA as candidate marker of late-stage clear cell RCC (12). However, further studies are still warranted to determine the mechanism of tumorgenesis and progression with aberrant ALDOA expression in RCC.

ALDOA has been reported to promote epithelial-mesenchymal transition (EMT) and migration in lung squamous 
cell carcinoma (8). EMT has been widely demonstrated to contribute to cancer dissemination and progression. EMT causes downregulation of epithelial markers, most notably E-cadherin, and upregulation of mesenchymal markers, such as $\mathrm{N}$-cadherin and vimentin (13). Kidney organogenesis involves mesenchymal-epithelial transition from original mesenchymal cells and formation of renal vesicles and tubules and maturation of nephrons (14). In RCC, this transition reverses, leading to EMT and dedifferentiation. The Wnt/ $\beta$-catenin signaling pathway is one of the major signaling pathways involved in RCC (15). $\beta$-catenin, a transcriptional coactivator, emerges as a key molecule in canonical Wnt signaling. Caspi et al reported that ALDOA regulates the Wnt signaling pathway (16).

In the present study, we aimed to determine the expression and function of ALDOA in RCC tissues and cells and its possible mechanism utilizing small interfering RNA (siRNA) and overexpression plasmids. Our results revealed that downregulated ALDOA expression could affect aberrant expression of E-cadherin, $\mathrm{N}$-cadherin, and vimentin in RCC cells and inactivate the $\mathrm{Wnt} / \beta$-catenin signaling pathway. These observations revealed that ALDOA may be a novel biomarker and provide a potential therapeutic strategy for treatment of RCC.

\section{Materials and methods}

Patients and tissue microarray (TMA). A total of 139 RCC tissues were obtained from patients who were treated by radical nephrectomy or partial nephrectomy between February 2008 and May 2011 at the First Affiliated Hospital of Nanjing Medical University (Nanjing, China). Pathologists confirmed identification of tumor tissues. None of the patients had been treated by either radiotherapy or chemotherapy. This study was approved by the Medical Ethics Committee of the hospital. Table I summarizes clinical and pathological features of 139 patients.

TMAs were constructed using the aforementioned 139 RCC tissues. All tissues were pathologically confirmed as RCC. An experienced pathologist reviewed hematoxylin and eosin slides again. Representative areas of specimens were identified and marked. Tissue cores measuring $2 \mathrm{~mm}$ from marked areas were selected from donor blocks and transferred to recipient paraffin blocks of TMA. Paraffin blocks were sectioned to produce serial $4-\mu \mathrm{m}$ sections. Then, immunohistochemical studies were performed on positively-charged slides mounted with $4 \mu \mathrm{m}$ of TMA paraffin blocks.

Immunohistochemistry. Sections from TMA paraffin blocks were removed from the incubator and successively dewaxed in xylene I and xylene II for $10 \mathrm{~min}$. Subsequently, the slides were rehydrated with sequential ethanol washes, starting at $100 \%$, followed by $95 \%, 85 \%$, and $75 \%$, and then incubated for $10 \mathrm{~min}$ in $3 \% \mathrm{H}_{2} \mathrm{O}_{2}$ to block endogenous peroxidase. Antigen retrieval was performed for $10 \mathrm{~min}$ by a steam pressure cooker containing citrate buffer. Then, the samples were blocked with $5-10 \%$ animal serum for $10 \mathrm{~min}$ and incubated with the appropriate antibody against ALDOA monoclonal antibody (1:100; cat. no. H00000226-M02; Abnova, Tapei, Taiwan) at $37^{\circ} \mathrm{C}$ for $2 \mathrm{~h}$ and $4^{\circ} \mathrm{C}$ overnight. After $5 \mathrm{~min}$ of washing with phosphate-buffered saline (PBS) thrice, slides were cultured in peroxidase-conjugated goat anti-mouse $\operatorname{IgG}(\mathrm{H}+\mathrm{L})$ secondary antibody (1:1,000; cat. no. ZB-2305; ZSGB-BIO, Inc., Beijing, China) for $20 \mathrm{~min}$. After two 5-min washes in PBS, reactions were visualized with a fresh substrate solution containing 3,3'-diaminobenzidine. Sections were counterstained with hematoxylin, dehydrated and dewaxed. Slides were sealed and analyzed by optical microscopy.

Evaluation of staining. Evaluation of protein staining was performed independently by two experienced pathologists who had no knowledge of obtained clinical and pathological data. Results of immunostaining for ALDOA were determined according to a previously described scoring system $(17,18)$. Percentages of positive tumor cells were estimated in at least five areas at an $\times 400$ magnification and assigned with one of the following quantitative scores: $0,0-5 \% ; 1,6-25 \% ; 2,26-50 \%$; $3,51-75 \%$; and $4,76-100 \%$. Intensity of positive staining was scored as follows: 1, low; 2, moderate; and 3, strong. Finally, a total score (0-12) for each sample was calculated by multiplying the quantitative score by the intensity score. Scores 0 to 4,5 to 8 , and 9 to 12 indicated negative (-), positive (+), and strong positive $(++)$ expression of ALDOA, respectively.

Cell culture and tissue samples. Human RCC cell lines (769-P, 786-0, ACHN and Caki-1) and normal renal proximal tubular cells (HK-2) were purchased from the Cell Bank Type Culture Collection of the Chinese Academy of Sciences (Shanghai, China). Cells of 769-P and 786-0 lines were maintained in Roswell Park Memorial Institute (RPMI)-1640 (Gibco; Thermo Fisher Scientific, Inc., Waltham, MA, USA), ACHN and HK-2 cells were maintained in Dulbecco's modified Eagle's medium, and Caki-1 was cultured in McCoy's 5A (both from Gibco; Thermo Fisher Scientific, Inc.). All media were supplemented with $10 \%$ fetal bovine serum (FBS; Gibco; Thermo Fisher Scientific, Inc.) within a humidified atmosphere containing $5 \% \mathrm{CO}_{2}$ at $37^{\circ} \mathrm{C}$.

Following the Local Ethics Committee of the First Affiliated Hospital of Nanjing Medical University (Nanjing, China), 21 paired tumor specimens and normal tissue samples for the detection of ALDOA expression were obtained with informed consent from RCC patients who had undergone radical nephrectomy or partial nephrectomy at the Department of Urology of the First Affiliated Hospital of Nanjing Medical University (Nanjing, China). Fresh samples were obtained during surgery, immediately frozen in liquid nitrogen, and stored at $-80^{\circ} \mathrm{C}$ until further analysis. Identification of tumor tissues and adjacent normal tissues was confirmed by histopathological examination.

Cell transfection. Cells from 786-0, Caki-1 and 769-Plines were seeded at a density of $1 \times 10^{6}$ cells/well in 6 -well plates at $70 \%$ confluence on the day before transfection. 786-0 and Caki-1 cell transfection was performed with Lipofectamine 2000 (Invitrogen; Thermo Fisher Scientific, Inc.) according to the manufacturer's instructions. Cells grown in 6-well plates were transfected with $100 \mathrm{pM}$ of synthetic ALDOA siRNA or negative control (NC). 769-P cells were transfected with ALDOA overexpression plasmids or vector controls using DNAfectin $^{\text {TM }}$ Plus (Applied Biological Materials (ABM), Richmond, BC, Canada), according to the manufacturer's instructions. Cells overexpressing ALDOA were defined as 
Table I. Association between ALDOA expression and clinicopathological factors in 139 RCC patients.

\section{ALDOA expression}

Variables Negative (-) Positive (+) Strong positive (++) P-value

n Proportion $(\%)$

Sex

Male

Female

Age, median (range), years

Side

Left

Right

Surgical procedure

Partial nephrectomy

Radical nephrectomy

Pathological type

Clear cell renal cell carcinoma

Papillary carcinoma

Chromophobe renal cell carcinoma

Other

Maximum diameter of the tumor, $\mathrm{cm}$

$\leq 4$

$>4-7$

$>7-10$

$>10$

Metastasis

Yes

No

Histological grade

Highly differentiated

Moderately differentiated

Poorly differentiated

$\begin{array}{rrr}6 & 41 & 37 \\ 11 & 13 & 31 \\ 55(37-75) & 55(20-86) & 57(27-81) \\ 9 & 24 & 36 \\ 8 & 30 & 32 \\ & & 21 \\ 2 & 11 & 47 \\ 15 & 43 & 56 \\ & & 5 \\ 17 & 53 & 6 \\ 0 & 0 & 1 \\ 0 & 1 & \\ 0 & 0 & 35 \\ & & 23 \\ 10 & 25 & 3 \\ 5 & 22 & 12 \\ 1 & 7 & 56 \\ 1 & 0 & \end{array}$

37

31

36

32

21

47

0.004

$\begin{array}{ll} & 84 \\ & 55 \\ & 55 \\ 0.763 & 56 \\ 0.621 & \end{array}$

60.4

$55 \quad 39.6$

56

\section{9}

70

49.6

50.4

0.175

$34 \quad 24.5$

$105 \quad 75.5$

0.026

$\begin{array}{rr}126 & 90.6 \\ 5 & 3.6 \\ 7 & 5.0 \\ 1 & 0.7\end{array}$

0.452

$70 \quad 50.4$
50

$50 \quad 36.0$

$15 \quad 10.8$

$4 \quad 2.9$

$\mathbf{0 . 0 2 0}$

$\begin{array}{rr}15 & 10.8 \\ 124 & 89.2\end{array}$

$\mathbf{0 . 0 3 3}$

$\begin{array}{rr}102 & 73.4 \\ 31 & 22.3 \\ 6 & 4.3\end{array}$

Bold, $\mathrm{P}<0.05$.

Table II. The sequences of siRNA and NC.

\begin{tabular}{lll}
\hline Name & & Sequences \\
\hline ALDOA-siRNA-1 & Sense & 5'-GCCUUGCCUGUCAAGGAAATT-3' \\
& Anti-sense & 5'-UUUCCUUGACAGGCAAGGCTT-3' \\
ALDOA-siRNA-2 & Sense & 5'-GCGUUGUGUGCUGAAGAUUTT-3' \\
& Anti-sense & 5'-AAUCUUCAGCACACAACGCTT-3' \\
ALDOA-siRNA-3 & Sense & 5'-GCCAGUAUGUGACCGAGAATT-3' \\
& Anti-sense & 5'-UUCUCGGUCACAUACUGGCTT-3' $^{\prime}$ \\
Negative control (NC) & Sense & 5'-UUCUCCGAACGUGUCACGUTT-3' \\
& Anti-sense & 5'-ACGUGACACGUUCGGAGAATT-3' \\
\hline
\end{tabular}

the OV group, while cells transfected with the vector alone were defined as the NC group. Six hours post-transfection with siRNA or NC and $16 \mathrm{~h}$ post-transfection with overexpression plasmids or vector controls, culture medium was replaced with
RPMI-1640 or McCoy's 5A containing FBS. Table II contains the sequences of ALDOA siRNA and NC. All used siRNA and $\mathrm{NC}$ were designed and synthesized by Shanghai GenePharma Co., Ltd,.., (Shanghai, China). ALDOA overexpression plasmids 
were constructed and purchased from Obio Technology Ltd. (Shanghai, China). Total RNA was collected $24 \mathrm{~h}$ after transfection and used for reverse transcription-polymerase chain reaction (RT-PCR) analysis to evaluate ALDOA expression. Total protein was prepared $60 \mathrm{~h}$ after transfection and used for western blot analysis. Other parts of the cells were used for cell proliferation, cell cycle, cell colony formation, and cell migration and invasion assays.

RNA isolation and RT-PCR. Total RNA was isolated from tissue samples or cultured cell lines by using TRIzol (Invitrogen; Thermo Scientific, Inc.) according to the manufacturer's instructions. Total RNA was reverse-transcribed into cDNAs using PrimeScript ${ }^{\mathrm{TM}}$ RT Master Mix (Perfect Real-Time) (Takara Biotechnology, Co., Ltd., Dalian, China) in accordance with the manufacturer's instructions. Conditions of reverse transcription were as follows: $37^{\circ} \mathrm{C}$ for $15 \mathrm{~min}$, $85^{\circ} \mathrm{C}$ for $5 \mathrm{sec}$, and $4^{\circ} \mathrm{C}$ until the end of the procedure. RNA and cDNA concentrations were assessed by NanoDrop (Thermo Fisher Scientific, Inc.). ALDOA expression was analyzed by RT-PCR using SYBR Green assay in accordance with the manufacturer's instructions (Applied Biosystems; Thermo Fisher Scientific, Inc.). Relative expression of ALDOA was calculated using the $2^{-\Delta \Delta C t}$ method. The following primer sequences were used: ALDOA: forward 5'-CGGGAAGAAG GAGAACCTG-3' and reverse 5'-GACCGCTCGGAGTGTA CTTT-3'; and $\beta$-actin: forward 5'-ACTGGAACGGTGAAG GTGAC-3' and reverse 5'-AGAGAAGTGGGGTGGCTTTT-3' (synthesized by Invitrogen, Shanghai, China). RT-PCR was performed under the following conditions: $50^{\circ} \mathrm{C}$ for $2 \mathrm{~min}$, $95^{\circ} \mathrm{C}$ for $2 \mathrm{~min} ; 40$ cycles at $95^{\circ} \mathrm{C}$ for $15 \mathrm{sec}$ and $60^{\circ} \mathrm{C}$ for $1 \mathrm{~min}$; and $95^{\circ} \mathrm{C}$ for $15 \mathrm{sec}, 60^{\circ} \mathrm{C}$ for $1 \mathrm{~min}$ and $95^{\circ} \mathrm{C}$ for $15 \mathrm{sec}$. Reactions were performed and analyzed by Applied Biosystems StepOne Plus Real-Time PCR System (Applied Biosystems; Thermo Fisher Scientific, Inc.). All reactions were run in triplicate.

Cell proliferation assay. Cells of 786-0, Caki-1 and 769-P lines were transfected to investigate the influence of ALDOA on the proliferative capacity of RCC cells. After $48 \mathrm{~h}$ of transfection, the cells were seeded onto 96 -well plates at a density of $2 \times 10^{3}$ cells/well and cultured for $24,48,72$ and $96 \mathrm{~h}$. Cell proliferation was determined using Cell Counting Kit-8 (CCK-8; Dojindo Molecular Technologies, Kumamoto, Japan) following the manufacturer's protocol. Absorbance was detected at an optical density (OD) of $450 \mathrm{~nm}$ by a spectrophotometer. In each group, three wells were assessed at each time-point.

Cell cycle assay. For the cell cycle analysis, $1 \times 10^{5}$ cells were harvested $48 \mathrm{~h}$ after transfection, washed twice with ice-cold PBS, and fixed with $70 \%$ ethanol at $-20^{\circ} \mathrm{C}$ overnight. Cells were incubated with $50 \mathrm{mg} / \mathrm{ml}$ of propidium iodide and $1 \mathrm{mg} / \mathrm{ml} \mathrm{RNase}$ for $30 \mathrm{~min}$ in the dark at room temperature. Using flow cytometry, treated cells were analyzed to determine distribution of cell cycle stages after transfection. At least 100,000 cells were necessary for each sample. Experiments were performed in triplicate.

Colony formation assay. To determine the long-term effects of ALDOA on cell colony formation, $48 \mathrm{~h}$ after transfection, the cells were seeded in culture dishes at a density of $1 \times 10^{3}$ cells/ dish and cultured at $37^{\circ} \mathrm{C}$ with medium exchange. Cells were grown for 10 days to form colonies contained more than 50 cells, which were stained with crystal violet, photographed, and counted under a fluorescence-inverted microscope (x100 magnification).

Cell migration and invasion assays. To investigate the possible effects of ALDOA on metastasis of RCC cells, migration and invasion assays were performed after transfection for $48 \mathrm{~h}$. For the migration assays, $2 \times 10^{4}$ cells in $200 \mu \mathrm{l}$ of serum-free medium were placed in the upper chamber of the Transwell (pore size, $8 \mu \mathrm{m}$; BD Biosciences, San Jose, CA, USA). For the invasion assays, $5 \times 10^{4}$ cells in $200 \mu \mathrm{l}$ of serum-free medium were placed in the upper chamber coated with Matrigel (BD Biosciences) in accordance with the manufacturer's protocol. The lower chamber was filled with $500 \mu \mathrm{l}$ of media containing $20 \%$ FBS. After incubating the cells for $24 \mathrm{~h}$ at $37^{\circ} \mathrm{C}$, the cells remaining in the upper membrane were removed by cotton swab, and those on the lower membrane surface were fixed in methanol and stained with crystal violet. Five random fields were photographed and counted under an optical microscope (x200 magnification). All experiments were performed in triplicate.

Protein isolation and western blot analysis. To further study the effects of ALDOA on protein changes in signaling pathways related to cell proliferation and metastasis in RCC cells, we isolated proteins from transfected cells and performed a western blot assay. Cells were washed thrice in PBS and lysed using radioimmunoprecipitation assay buffer (KeyGen Biotech Co., Ltd., Nanjing, Jiangsu, China) supplemented with protease inhibitors at $4^{\circ} \mathrm{C}$ for $30 \mathrm{~min}$. The protein concentration was measured using a BCA kit (Beyotime Institute of Biotechnology, Nantong, Jiangsu, China), according to the manufacturer's instructions. The same amounts of proteins were electrophoresed in $10 \%$ sodium dodecyl sulfate polyacrylamide gel, transferred to a polyvinylidene fluoride membrane (EMD Millipore, Billerica, MA, USA), blocked for $2 \mathrm{~h}$ with $5 \%$ non-fat milk at room temperature, and incubated with primary antibodies at $4^{\circ} \mathrm{C}$ overnight. Then, the membrane was incubated with horseradish peroxidase-conjugated secondary antibody for $2 \mathrm{~h}$ after washing thrice with Tris-buffered saline and $0.1 \%$ Tween-20. Antibodies against ALDOA (1:400; cat. no. H00000226-M02; Abnova, Tapei, Taiwan), actin (1:3,000; cat. no. ab179467; Abcam, Cambridge, UK), E-cadherin (1:1,000; cat. no. 3195), N-cadherin (1:1,000; cat. no. 13116), vimentin (1:1,000; cat. no. 5741), $\beta$-catenin (1:1,000; cat. no. 8480), phospho- $\beta$-catenin $(1: 1,000$; cat. no. 9567), cyclin D1 (1:1,000; cat. no. 2978), Met (1:1,000; cat. no. 8198), matrix metalloproteinase-7 (MMP-7) (1:1,000; cat. no. 3801) and c-Myc (1:1,000; cat. no. 5605) (Cell Signaling Technology, Danvers, MA, USA) were used in western blot analysis in accordance with the manufacturer's instructions. Blots were detected using enhanced chemiluminescence (Thermo Fisher Scientific, Inc.). The protein levels were determined by normalization to actin.

Statistical analyses. SPSS Statistics 20 software package (IBM Corp. Armonk, NY, USA) and GraphPad Prism 5 
A

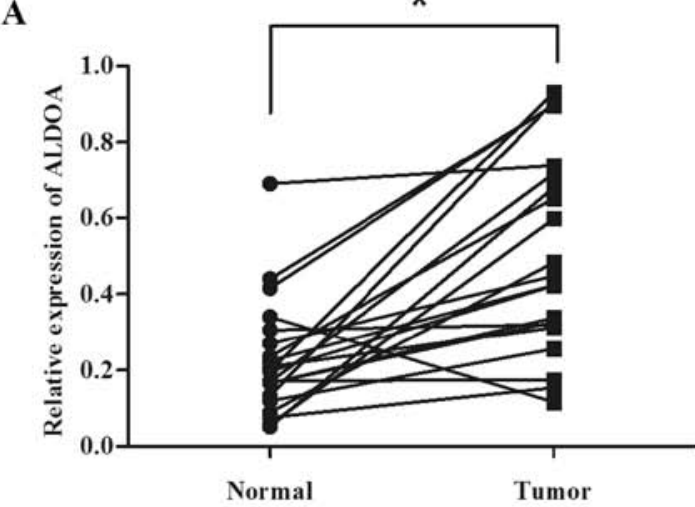

C

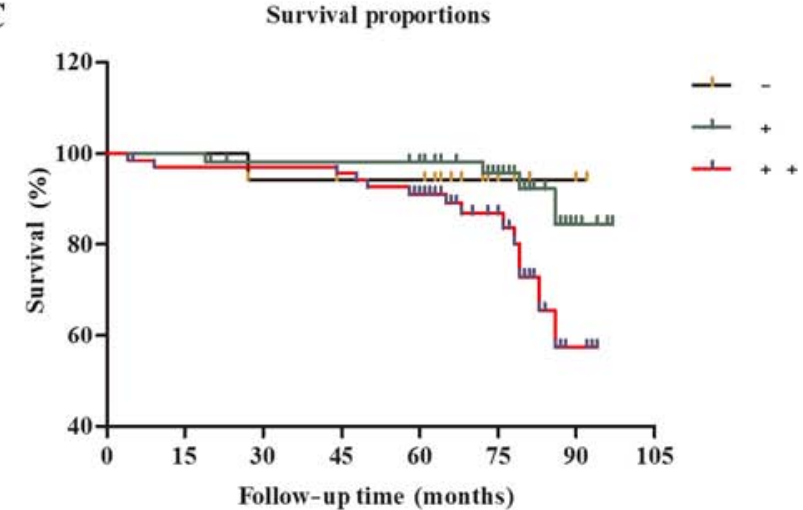

B

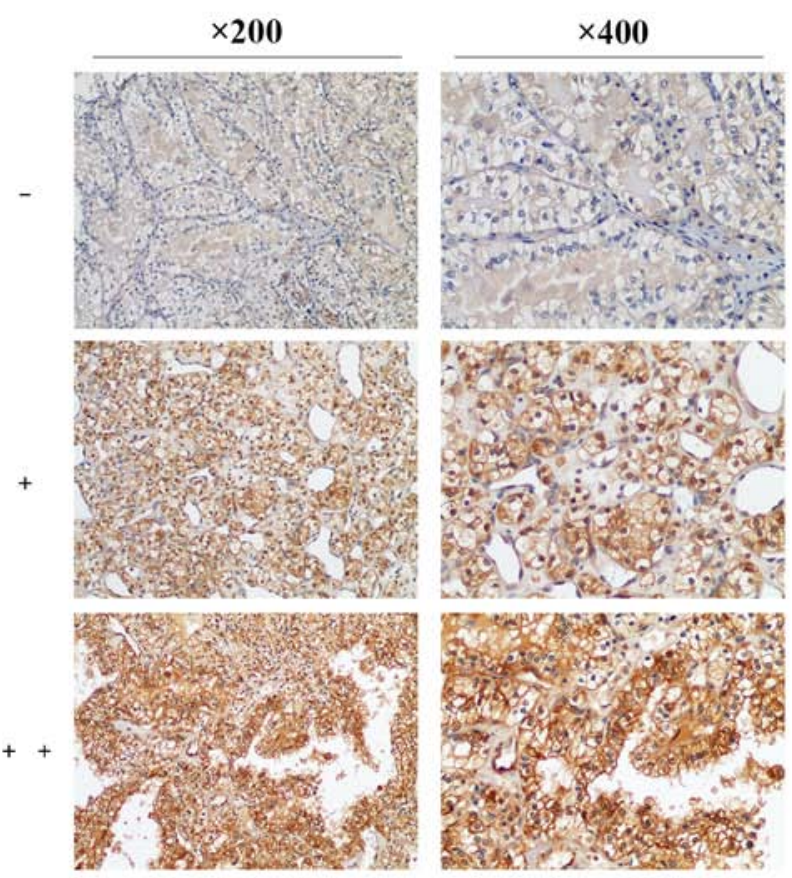

Figure 1. High expression of ALDOA in RCC tumor tissues. (A) Compared with paired adjacent non-tumor tissues, RCC samples exhibited significantly upregulated ALDOA levels. The median in each triplicate result was used to calculate the relative ALDOA concentration by using the comparative $2^{-\Delta C t}$ method. ${ }^{*} \mathrm{P}<0.05$ compared with the non-tumor tissues. (B) Expression of ALDOA was assayed by immunohistochemical staining. (-), negative; (+), positive; $(++)$, strongly positive. (C) Univariate Kaplan-Meier/log-rank (Mantel-Cox) analysis of survival vs. ALDOA expression. ALDOA, fructose-bisphosphate aldolase A; RCC, renal cell carcinoma.

(GraphPad Software, Inc., La Jolla, CA, USA) were used for statistical analysis. We used Chi-square test to analyze the relationship between ALDOA expression and clinicopathological factors. Univariate survival was assessed using the Kaplan-Meier curve and log-rank test. Results are presented as the mean \pm standard deviation (SD). Differences between two groups were analyzed using Student's t-test. $\mathrm{P}<0.05$ was considered to indicate a statistically significant difference.

\section{Results}

High expression of ALDOA in RCC tumor tissues. To detect ALDOA expression pattern in RCC, we first examined mRNA levels of ALDOA in 21 patients diagnosed with the disease. RT-PCR was performed to investigate ALDOA expression in 21 paired RCC tissues and adjacent non-tumor tissues. Compared with paired adjacent non-tumor tissues, RCC samples presented significantly upregulated ALDOA levels $(20 / 21)(\mathrm{P}<0.0001$; Fig. 1A). To investigate the presence of similar changes at the translational level, we determined ALDOA protein expression in 139 cases of RCC. The TMA of 139 RCC tissues and immunohistochemistry staining revealed that percentages of positive and strong positive ALDOA expression reached $38.8 \%$ (54/139) and 48.9\% (68/139), respectively (ALDOA expression rate $=87.8 \%$, Fig. 1B).

Next, we used the Chi-square test to evaluate the relationship of ALDOA expression and clinicopathological factors of
RCC. The results revealed the absence of a significant difference between the ALDOA expression and constituent ratio in the different groups of the maximum diameter of the tumor $(\mathrm{P}=0.452)$ (Table I). However, ALDOA expression was significantly associated with metastasis $(\mathrm{P}=0.020)$ and histological grade $(\mathrm{P}=0.033)$ (Table I).

Kaplan-Meier analysis of patient data revealed notably shorter overall survival time of higher ALDOA-expressing individuals than those with lower ALDOA expression $(\mathrm{P}=0.0341)$ (Fig. 1C). The overall survival rates of the negative, positive and strong positive ALDOA expression groups were 94.1, 92.6 and 79.4\%, respectively. Thus, high expression of ALDOA in RCC patients contributed to advanced RCC and poorer survival. Then, we carried out research on the biological roles of ALDOA in RCC cell lines to further study the aforementioned findings.

ALDOA is upregulated in RCC cell lines. The expression level of ALDOA was determined in four RCC cell lines (769-P, 786-0, ACHN and Caki-1) and in normal renal proximal tubular cell line (HK-2) by RT-PCR and western blot analysis. Expression of ALDOA was significantly higher in RCC cell lines than in HK-2 cells $(\mathrm{P}<0.01$; Fig. $2 \mathrm{~A}$ and $\mathrm{B})$. Among the four RCC cell lines, 786-0 and Caki-1 exhibited the highest ALDOA level and 769-P exhibited the lowest ALDOA level. Thus, 786-0, Caki-1 and 769-P were selected for subsequent cell functional experiments. 
A

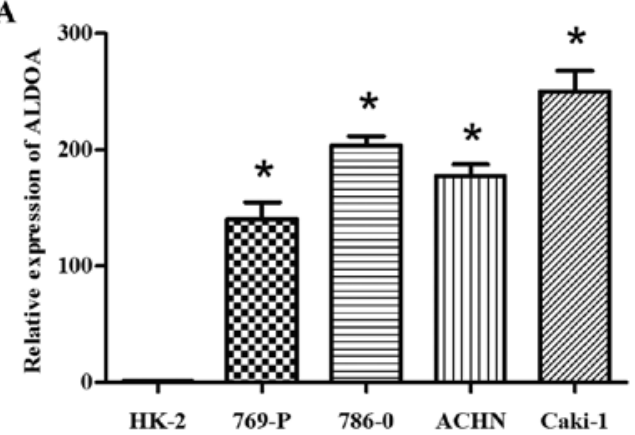

B

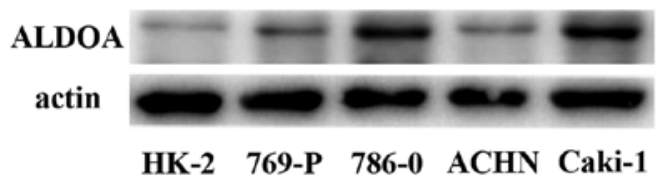

C

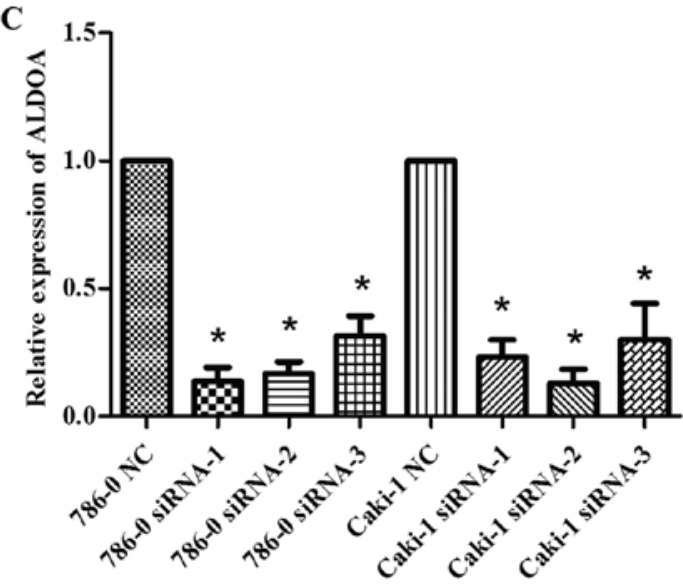

D

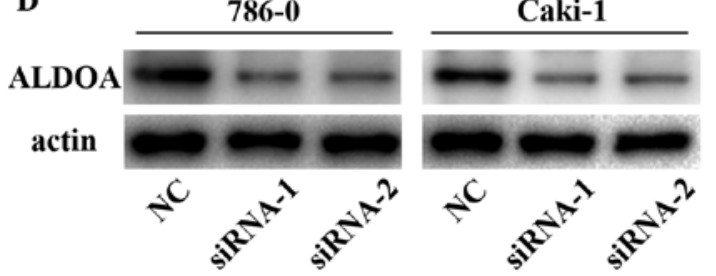

E

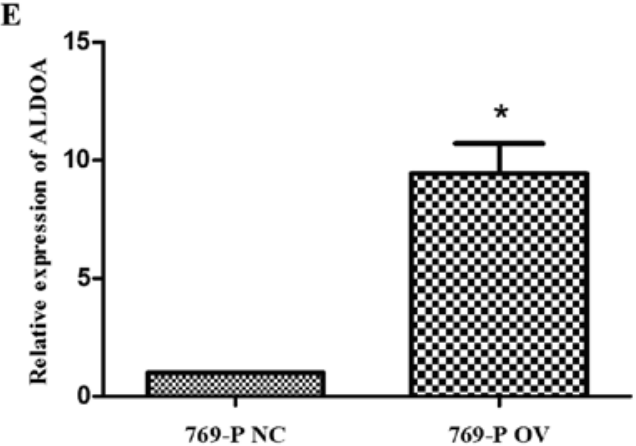

F

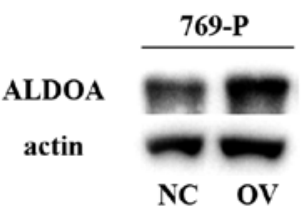

Figure 2. ALDOA is upregulated in RCC cell lines. (A) Compared with normal renal cell line HK-2, RCC cell lines exhibited relatively high expression levels of ALDOA. The median in each triplicate result was used to calculate the relative ALDOA concentration by using the comparative $2^{-\Delta \Delta C t}$ method. ${ }^{*} \mathrm{P}<0.05$ compared with the normal cell line. (B) ALDOA protein expression in RCC cell lines and a normal renal cell line. Actin was used as an internal control. (C) ALDOA mRNA expression after $24 \mathrm{~h}$ of transfection with ALDOA-siRNA or NC. The median in each triplicate result was used to calculate the relative ALDOA concentration using comparative the $2^{-\Delta \Delta C t}$ method. ${ }^{*} \mathrm{P}<0.05$ compared with NC. (D) ALDOA protein expression after $48 \mathrm{~h}$ of transfection with ALDOA-siRNA or NC in 786-0 and Caki-1 cells. Actin was used as an internal control. (E) ALDOA mRNA expression after $24 \mathrm{~h}$ of transfection with ALDOA overexpression plasmids or vector controls. The median in each triplicate result was used to calculate the relative ALDOA concentration using the comparative $2^{-\Delta \triangle C t}$ method. "P $<0.05$ compared with NC. (F) ALDOA protein expression after $48 \mathrm{~h}$ of transfection with ALDOA overexpression plasmids or vector controls in 769-P cells. Actin was used as an internal control.

To investigate the effects of ALDOA in RCC cells, we knocked down ALDOA expression by transfecting siRNA and overexpressed ALDOA by transfecting overexpression plasmids. ALDOA expression in RCC samples was significantly lower with ALDOA-siRNA transfection than that in $\mathrm{NC}(\mathrm{P}<0.05$; Fig. $2 \mathrm{C}$ and $\mathrm{D})$ and markedly upregulated after ALDOA overexpression plasmid transfection $(\mathrm{P}=0.008$; Fig. 2E and F). Due to more efficient transfection we used the first and second ALDOA-siRNA in the following experiments. was performed to assess cell proliferation in 786-0, Caki-1 and
769-P cells after transfection. Compared with the NC group, a significant difference was detected at 72 and $96 \mathrm{~h}$ in $786-0$ and 769-P cells and at $96 \mathrm{~h}$ in Caki-1 cells $(\mathrm{P}<0.05$; Fig. 3A). Flow cytometric analysis was performed to explore the effects of inhibition on cell proliferation. Analysis revealed remarkably higher percentages of 786-0 and Caki- 1 cells transfected with ALDOA-siRNA in the G0/G1 phase than those transfected with NC siRNA, indicating that ALDOA can induce G0/G1 cell cycle arrest in RCC cells $(\mathrm{P}<0.05$; Fig. 3B).

ALDOA promotes colony formation of RCC cells. Colony formation was observed after transfection with ALDOA-siRNA 

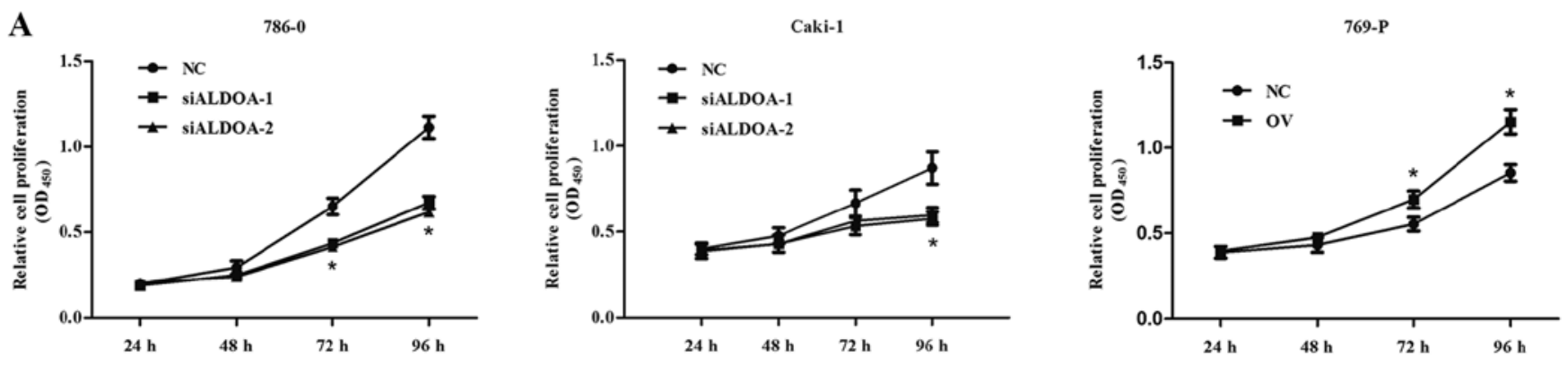

B
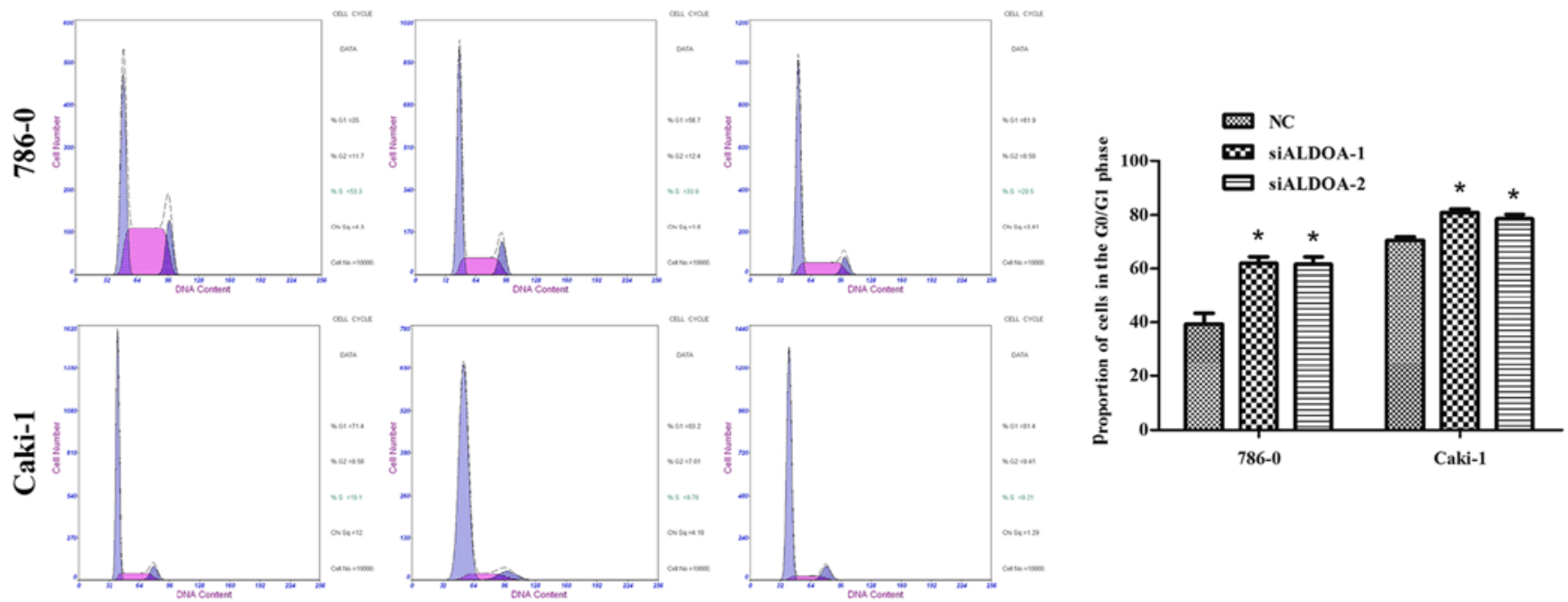

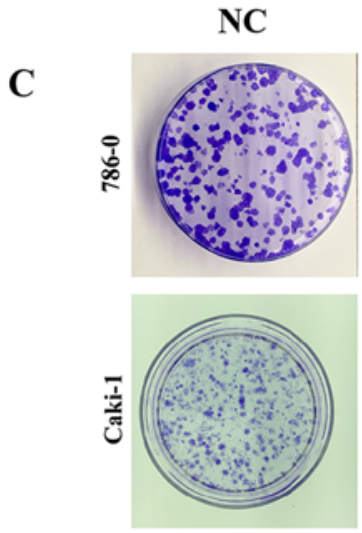

NC
SiALDOA-1

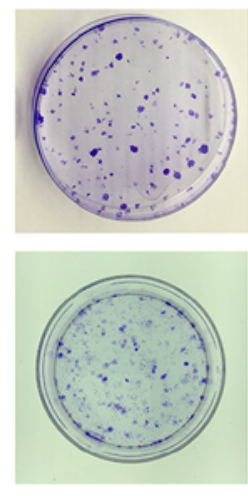

siALDOA-1

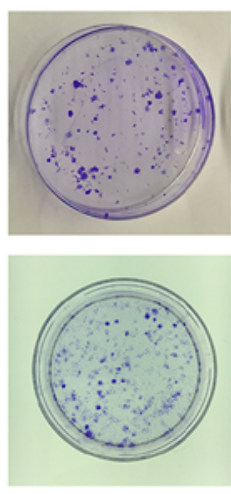

SiALDOA-2

SiALDOA-2

Figure 3. ALDOA promotes cell proliferation and colony-forming potential in RCC cell lines. (A) Assessment of cell proliferation was performed using CCK-8 assay. Downregulation of ALDOA significantly inhibited proliferation of 786-0 and Caki-1 cells ( $\left.{ }^{*} \mathrm{P}<0.05\right)$. Overexpression of ALDOA significantly promoted proliferation of 769-P cells ( $\left.{ }^{*} \mathrm{P}<0.05\right)$. (B) Flow cytometric analysis revealed that after downregulation of ALDOA, the cell cycle of 786-0 and Caki-1 cells was arrested at the G1 phase. The histogram indicates the percentage of cells in the G1 phase $\left({ }^{*} \mathrm{P}<0.05\right)$. (C) Downregulation of ALDOA inhibited colony formation in $\mathrm{RCC}$ cell lines. Data represent the mean $\pm \mathrm{SD}$ of at least three independent experiments $\left({ }^{*} \mathrm{P}<0.05\right)$.

and NC siRNA. Compared with the NC group, downregulation of ALDOA significantly inhibited colony formation in 786-0 and Caki-1 cell lines ( $\mathrm{P}<0.001$; Fig. 3C).

ALDOA promotes migration and invasion in RCC cells. We used Transwell assays to detect changes in migration and invasion capabilities after knocking down and upregulating ALDOA expression. The migration assay indicated that downregulation of ALDOA significantly suppressed migration capability in 786-0 and Caki-1 cells compared with NC $(\mathrm{P}<0.05$; Fig. 4A). Similarly, the invasion assay indicated that downregulation of ALDOA inhibited invasion capability in
786-0 and Caki-1 cells compared with NC ( $\mathrm{P}<0.01$; Fig. 4B). Conversely, overexpression of ALDOA enhanced the migration and invasion of 769-P cells $(\mathrm{P}<0.001$; Fig. $4 \mathrm{C}$ and $\mathrm{D})$. These results revealed that ALDOA may play an important role in RCC progression.

ALDOA promotes EMT in RCC cells. As aforementioned, EMT is a key process in RCC progression and metastasis. Considering the effect of ALDOA on RCC cell migration and invasion, we further investigated whether ALDOA could affect EMT markers in RCC cells using western blot analysis. The results revealed a gain in E-cadherin expression in Caki-1 cells 
A
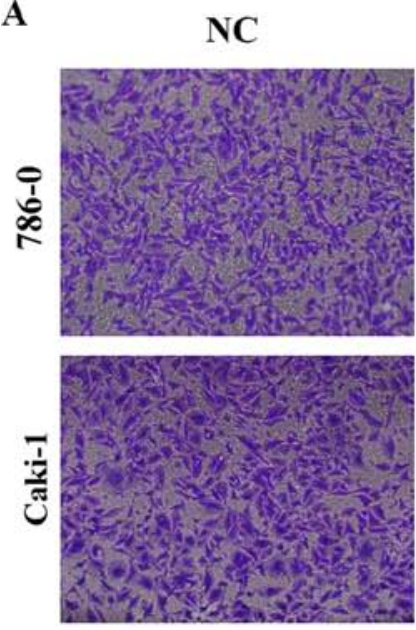

B
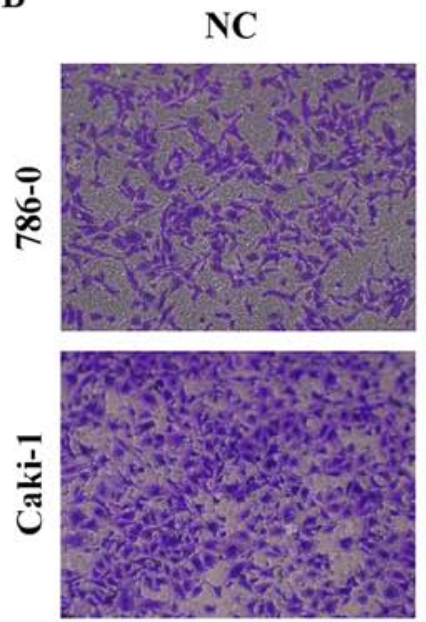

C
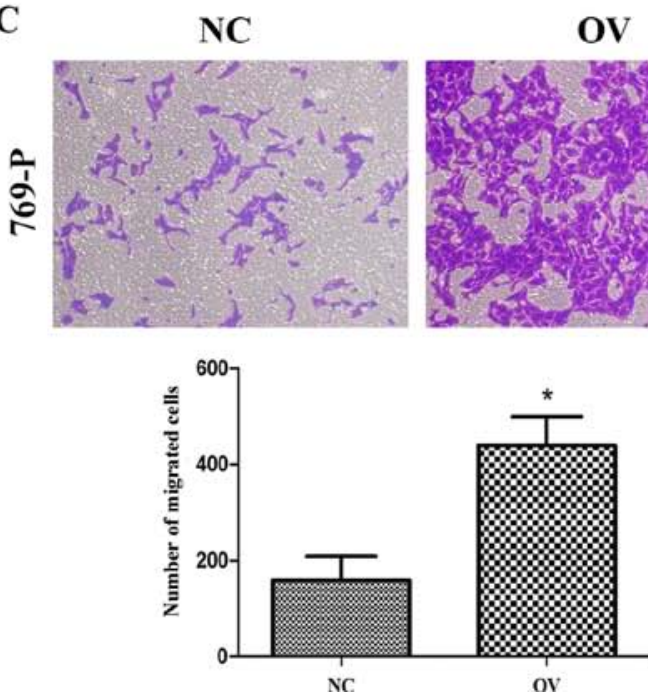

SiALDOA-2
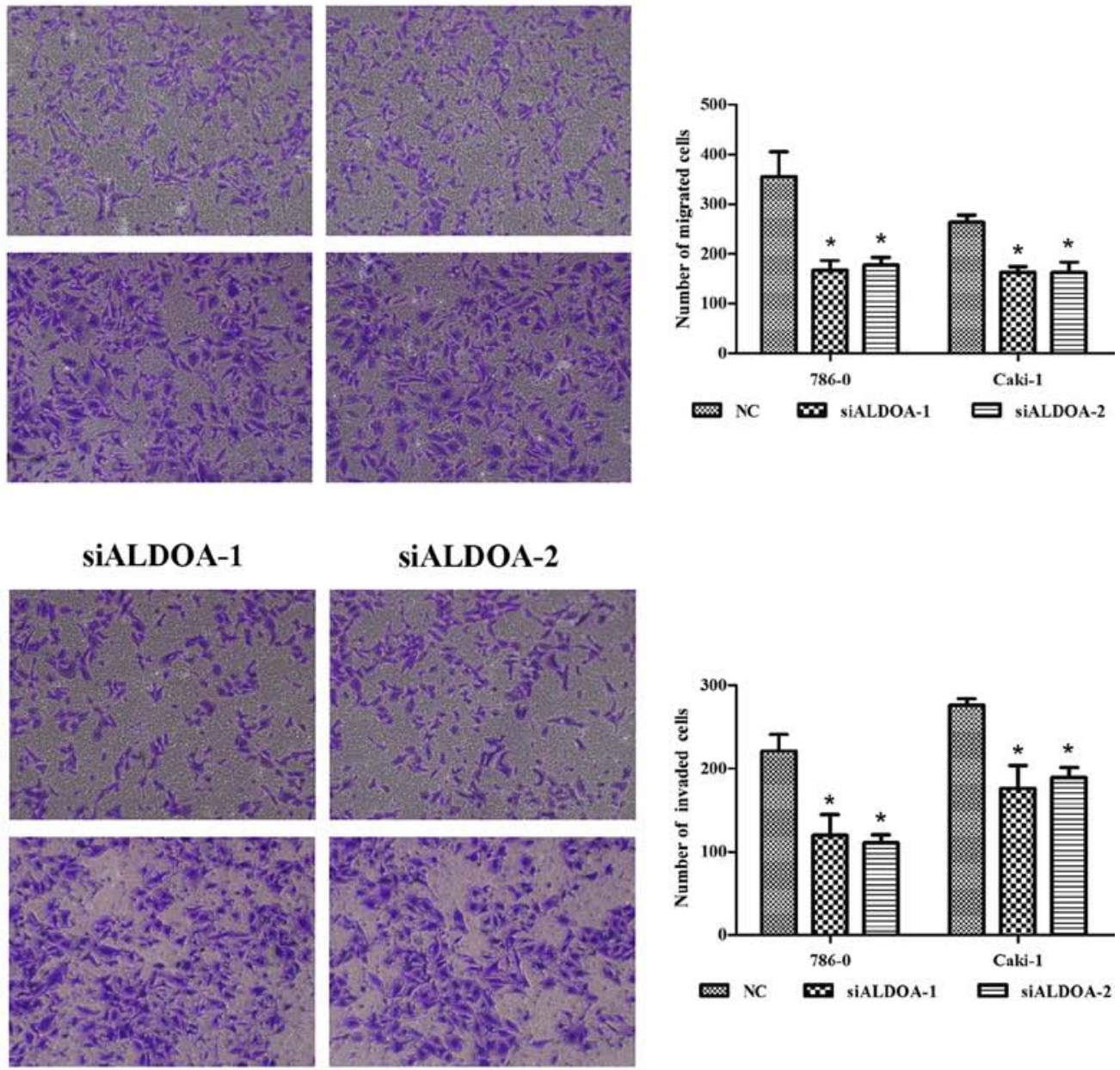

D
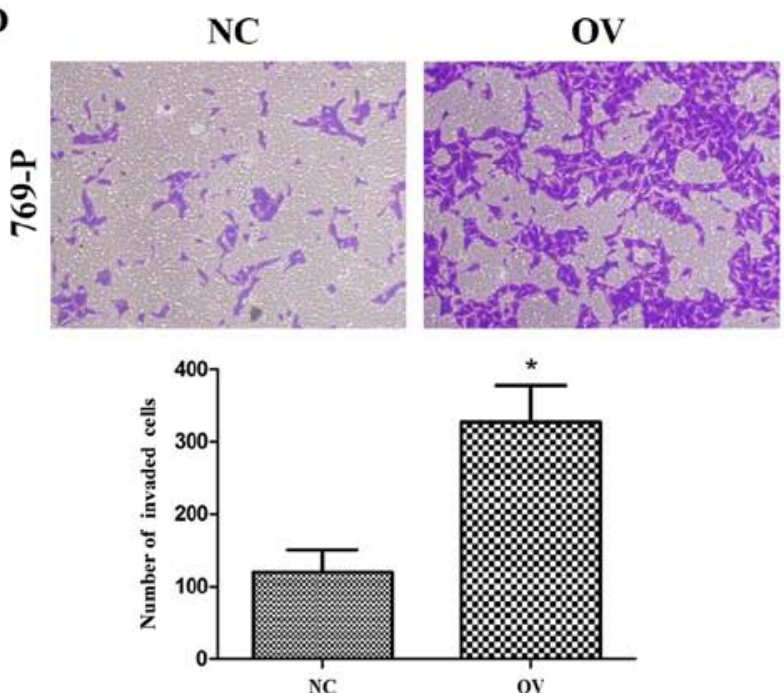

Figure 4. ALDOA promotes cell migration and invasion in RCC cell lines. (A and B) Downregulation of ALDOA inhibited migration and invasion in RCC cell lines. (C and D) Upregulation of ALDOA enhanced migration and invasion in RCC cell lines. Data represent the mean \pm SD of at least three independent experiments $($ ( $\mathrm{P}<0.05)$. Original magnification, $\mathrm{x} 200$.

and loss of N-cadherin and vimentin in both 786-0 and Caki-1 after transfection with ALDOA-siRNA (Fig. 5A), suggesting that ALDOA induced EMT.

ALDOA may activate the Wnt/ $\beta$-catenin signaling pathway. Our aforementioned results revealed that ALDOA promoted
RCC cell proliferation, colony formation, migration and invasion, and EMT. Thus, to assess the mechanism by which ALDOA contributed to RCC progression, we examined the effect of transient ALDOA downregulation on the $\mathrm{Wnt} / \beta$-catenin signaling pathway, which is involved in tumor development. Although no difference was detected 
A

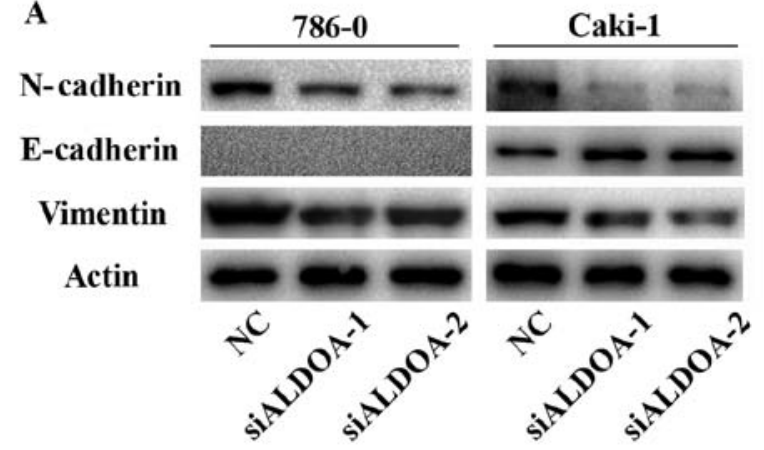

B

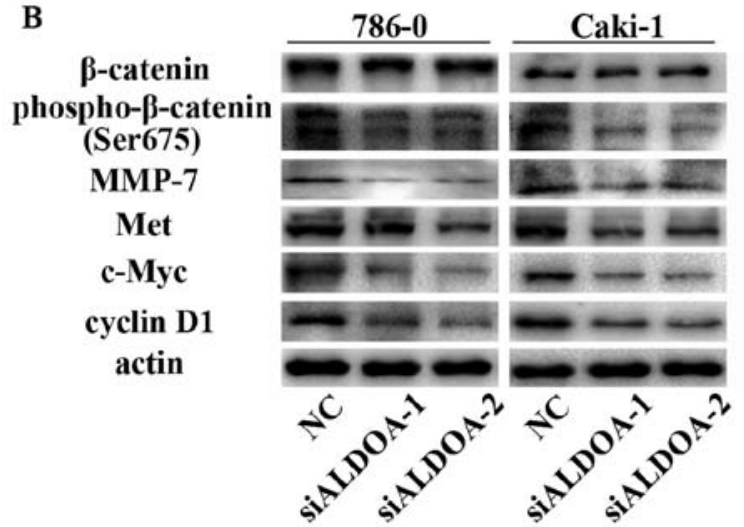

Figure 5. ALDOA may induce RCC progression via EMT and the Wnt/ $\beta$-catenin signaling pathway. (A) We used western blot analysis to detect changes in EMT markers in 786-0 and Caki-1 cells transfected by ALDOA-siRNA. A gain in E-cadherin expression and loss of N-cadherin and vimentin were observed in treated cells. Actin was used as a loading control. (B) Western blot analysis was used to detect changes in the protein

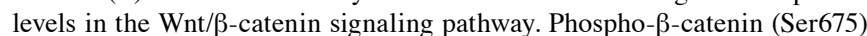
MMP-7, Met, c-Myc, and cyclin D1 levels were decreased with downregulation of ALDOA, but no changes were detected in the expression of total $\beta$-catenin. Actin was used as a loading control.

in total $\beta$-catenin expression, phospho- $\beta$-catenin (Ser675) was effectively reduced in ALDOA-siRNA-transfected RCC cells (Fig. 5B). Downstream target proteins, such as MMP-7, Met, c-Myc, and cyclin D1, were significantly decreased in ALDOA-downregulated RCC cells (Fig. 5B). All these findings revealed that ALDOA may induce cell proliferation and metastasis through the $\mathrm{Wnt} / \beta$-catenin signaling pathway.

\section{Discussion}

Given the improvement of people's health awareness and inspection technology, incidence of RCC, which is one of the common malignant tumors of the urinary system, has increased recently. However, in patients with localized RCC, probability of metastasis remains as high as $40 \%$; and tumor metastasis of these patients results in a median survival time of approximately 6-12 months, with a 5-year survival rate of 9\% (19). As radiotherapy and chemotherapy are insensitive to RCC, surgical resection remains the first choice for treatment (19). Therefore, in absence of other effective therapies, further studies on the mechanism of genesis and development of RCC and finding new biomarkers are necessary for promoting early diagnosis and treatment.

ALDOA participates not only in glycolysis but also in tumor development and affects prognosis with aberrant expression in some tumors $(8-10,20)$.In the present study, we first used RT-PCR to confirm high expression of ALDOA in RCC tissues and cells in comparison with normal ones, suggesting that increased expression of ALDOA may be related to the occurrence of RCC. Then, TMA and immunohistochemistry were performed to analyze 139 RCC tissue samples. Strong positive expression of ALDOA accounted for 48.9\% (68/139), positive expression reached $38.8 \%$ (54/139), and negative expression totaled $12.2 \%$ (17/139), further supporting high ALDOA expression in RCC, and this condition may be related to oncogenesis. Our clinical data indicated that patients with strong positive expression of ALDOA featured higher incidence of metastasis and worse histological differentiation. However, tumor size did not exhibit any association with ALDOA expression. During follow-up of the 139 RCC patients, Kaplan-Meier analysis revealed that negative ALDOA expression resulted in the longest survival among patients, whereas those with strong positive ALDOA expression exhibited the shortest survival period. All these results strongly suggest that ALDOA possesses clinical value as a prognostic factor for RCC.

Considering the confirmed high ALDOA expression in RCC cell lines by RT-PCR and western blot analysis, siRNA and overexpression plasmid transfection approaches were employed to investigate the potential biological effects of ALDOA on RCC cell function. Two independent siRNAs significantly decreased the expression of ALDOA in 786-0 and Caki-1 cells, and the overexpressed plasmid effectively upregulated ALDOA expression in 769-P cells. We observed that ALDOA knockdown significantly inhibited growth of 786-0 and Caki-1 cells, resulting from the arrest in the G0/G1 phase of the cell cycle. The G1 phase starts from mitosis to the period before DNA replication, in which RNA and ribosomes are synthesized. When arrested in the G1 phase, the cells remain in the G0 phase, where cell division stops, thus inhibiting cell proliferation. Western blot analysis revealed decreased expression of protein cyclin D1 in RCC cells transfected with ALDOA-siRNA compared with NC. Cyclin D1 is a critical target of proliferative signals in the G1 phase and is rapidly synthesized and accumulated in the nucleus in the G1 phase and disappears as cells proceed to the S phase (21). Overexpression of cyclin D1 is considered to be associated with early-stage cancer and progression (22). Thus, decreased cyclin D1 expression may induce arrest in the G1 phase after downregulating ALDOA expression. Combined with colony formation, we speculated that ALDOA may promote RCC cell proliferation. Previous studies reported that EMT, which plays an important role in cancer invasion and metastasis, induces physical translocation of cancer cells to distant organs and their development into metastatic lesions $(23,24)$. Our data indicated that ALDOA overexpression increased the migration and invasion abilities of RCC cells, while ALDOA knockdown significantly reduced these abilities by blocking EMT, downregulating the expression of $\mathrm{N}$-cadherin and vimentin in 786-0 and Caki-1 cells, and upregulating the expression of E-cadherin in Caki-1 cells. Coinciding with results reported in literature $(25,26)$, E-cadherin was not detected in $786-0$ cells. These findings revealed that ALDOA may function as a tumor promoter in RCC.

Recently, a research study reported that aldolase proteins can regulate novel Wnt signaling; for example, ALDOA 
activates Wnt signaling by disrupting glycogen synthase kinase 3 (GSK-3) and $\beta$-axin interaction and targeting axin to the dishevelled-induced signalosomes (16). The Wnt signaling pathway is involved in many cellular processes, including proliferation, migration, differentiation, movement, and survival (27). In unstimulated cells, the $\beta$-catenin destruction complex, which contains scaffolding protein axin, adenomatous polyposis coli protein, GSK-3 $\alpha / \beta$, and casein kinase- $1 \alpha$, maintains extremely low cytosolic and nuclear levels of $\beta$-catenin by promoting phosphorylation and ubiquitination of $\beta$-catenin $(28,29)$. In the present study, we revealed that ALDOA knockdown reduced the expression of phospho$\beta$-catenin (Ser675). Previous studies have demonstrated that $\beta$-catenin can be phosphorylated by protein kinase A (PKA) at site Ser675, and phosphorylation by PKA promotes transcriptional activity and binding of $\beta$-catenin to its transcriptional coactivator $(30,31)$. Thus, knockdown of ALDOA expression decreased transcriptional activity of $\beta$-catenin at the nuclear level. Expression of $\mathrm{Wnt} / \beta$-catenin-activated target proteins MMP-7, Met, c-Myc and cyclin D1 decreased after ALDOA knockdown. MMP-7, Met and c-Myc are important factors of tumor invasion, angiogenesis, carcinogenesis, and apoptosis (32-34), whereas cyclin D1 is associated with the cell cycle (22). Thus, we speculated that ALDOA may influence RCC progression through the $\mathrm{Wnt} / \beta$-catenin signaling pathway.

In summary, our results revealed that ALDOA was significantly upregulated in RCC. Positive ALDOA expression was associated with metastasis, histological differentiation, and prognosis of RCC patients. Silencing ALDOA expression in RCC cells by specific siRNA significantly decreased their proliferative, migratory, and invasive abilities, while ALDOA overexpression increased these abilities. ALDOA may serve as a potential tumor promoter in RCC by EMT and the Wnt/ $\beta$-catenin signaling pathway. Consequently, ALDOA may be a competent candidate target for the diagnosis and therapy of RCC. Further studies are still required to research the mechanism and assess the role of ALDOA in vivo in the future.

\section{Acknowledgements}

We would like to thank all the colleagues who helped us on designing and performing the experiments.

\section{Funding}

This study was supported by the National Natural Science Foundation of China (nos. 81372757 and 81570676) and the Science and Education Health Project of Jiangsu Province for important talent (no. RC2011055).

\section{Availability of data and materials}

The datasets used during the present study are available from the corresponding author upon reasonable request.

\section{Authors' contributions}

MG, ZW and XJ conceived and designed the study. ZH, YH, YT and MB performed the experiments. Analysis and inter- pretation of data was conducted by $\mathrm{ZH}, \mathrm{YL}$ and $\mathrm{SW}$. ZH and YT wrote the paper. ZH, CQ, JQ and QC reviewed and edited the manuscript. All authors read and approved the manuscript and agree to be accountable for all aspects of the research in ensuring that the accuracy or integrity of any part of the work are appropriately investigated and resolved.

\section{Ethics approval and consent to participate}

This study was approved by the Medical Ethics Committee of the First Affiliated Hospital of Nanjing Medical University and informed consent was obtained from the patients.

\section{Consent for publication}

Not applicable.

\section{Competing interests}

The authors declare that they have no competing interests.

\section{References}

1. Siegel RL, Miller KD and Jemal A: Cancer statistics, 2016. CA Cancer J Clin 66: 7-30, 2016.

2. Yang L, Parkin DM, Ferlay J, Li L and Chen Y: Estimates of cancer incidence in China for 2000 and projections for 2005. Cancer Epidemiol Biomarkers Prev 14: 243-250, 2005.

3. Chow WH, Dong LM and Devesa SS: Epidemiology and risk factors for kidney cancer. Nat Rev Urol 7: 245-257, 2010.

4. Kajita E, Moriwaki J, Yatsuki H, Hori K, Miura K, Hirai M and Shiokawa K: Quantitative expression studies of aldolase A, $\mathrm{B}$ and $\mathrm{C}$ genes in developing embryos and adult tissues of Xenopus laevis. Mech Dev 102: 283-287, 2001.

5. Yao DC, Tolan DR, Murray MF, Harris DJ, Darras BT, Geva A and Neufeld EJ: Hemolytic anemia and severe rhabdomyolysis caused by compound heterozygous mutations of the gene for erythrocyte/muscle isozyme of aldolase, ALDOA(Arg303X/ Cys338Tyr). Blood 103: 2401-2403, 2004.

6. Kishi H, Mukai T, Hirono A, Fujii H, Miwa S and Hori K: Human aldolase A deficiency associated with a hemolytic anemia: Thermolabile aldolase due to a single base mutation. Proc Natl Acad Sci USA 84: 8623-8627, 1987.

7. Miwa S, Fujii H, Tani K, Takahashi K, Takegawa S, Fujinami N, Sakurai M, Kubo M, Tanimoto Y, Kato T, et al: Two cases of red cell aldolase deficiency associated with hereditary hemolytic anemia in a Japanese family. Am J Hematol 11: 425-437, 1981.

8. Du S, Guan Z, Hao L, Song Y, Wang L, Gong L, Liu L, Qi X, Hou Z and Shao S: Fructose-bisphosphate aldolase a is a potential metastasis-associated marker of lung squamous cell carcinoma and promotes lung cell tumorigenesis and migration. PLoS One 9: e85804, 2014.

9. Ji S, Zhang B, Liu J, Qin Y, Liang C, Shi S, Jin K, Liang D, Xu W, $\mathrm{Xu} \mathrm{H}$, et al: ALDOA functions as an oncogene in the highly metastatic pancreatic cancer. Cancer Lett 374: 127-135, 2016.

10. Peng Y, Li X, Wu M, Yang J, Liu M, Zhang W, Xiang B, Wang X, $\mathrm{Li} \mathrm{X}, \mathrm{Li} \mathrm{G}$, et al: New prognosis biomarkers identified by dynamic proteomic analysis of colorectal cancer. Mol Biosyst 8: 3077-3088, 2012.

11. Takashi M, Zhu Y, Nakano Y, Miyake K and Kato K: Elevated levels of serum aldolase $A$ in patients with renal cell carcinoma. Urol Res 20: 307-311, 1992.

12. Neely BA, Wilkins CE, Marlow LA, Malyarenko D, Kim Y, Ignatchenko A, Sasinowska H, Sasinowski M, Nyalwidhe JO, Kislinger T, et al: Proteotranscriptomic analysis reveals stage specific changes in the molecular landscape of clear-cell renal cell carcinoma. PLoS One 11: e0154074, 2016.

13. He $\mathrm{H}$ and Magi-Galluzzi C: Epithelial-to-mesenchymal transition in renal neoplasms. Adv Anat Pathol 21: 174-180, 2014.

14. Rivera MN and Haber DA: Wilms' tumour: Connecting tumorigenesis and organ development in the kidney. Nat Rev Cancer 5: $699-712,2005$. 
15. Banumathy $G$ and Cairns P: Signaling pathways in renal cell carcinoma. Cancer Biol Ther 10: 658-664, 2010.

16. Caspi M, Perry G, Skalka N, Meisel S, Firsow A, Amit M and Rosin-Arbesfeld R: Aldolase positively regulates of the canonical Wnt signaling pathway. Mol Cancer 13: 164, 2014

17. Tong X, Li K, Luo Z, Lu B, Liu X, Wang T, Pang M, Liang B, Tan M, Wu M, et al: Decreased TIP30 expression promotes tumor metastasis in lung cancer. Am J Pathol 174: 1931-1939, 2009.

18. Cheng G, Wang S, Li X, Li S, Zheng Y, Zhang L, Bao M, Liang C, Huang Z, Liu Y, et al: Positive expression of NR6A1/CT150 as a predictor of biochemical recurrence-free survival in prostate cancer patients. Oncotarget 8: 64427-64439, 2016.

19. van Spronsen DJ, de Weijer KJ, Mulders PF and De Mulder PH Novel treatment strategies in clear-cell metastatic renal cell carcinoma. Anticancer Drugs 16: 709-717, 2005.

20. Long F, Cai X, Luo W, Chen L and Li K: Role of aldolase A in osteosarcoma progression and metastasis: In vitro and in vivo evidence. Oncol Rep 32: 2031-2037, 2014.

21. Baldin V, Lukas J, Marcote MJ, Pagano M and Draetta G: Cyclin D1 is a nuclear protein required for cell cycle progression in G1. Genes Dev 7: 812-821, 1993.

22. Diehl JA: Cycling to cancer with cyclin D1. Cancer Biol Ther 1: 226-231, 2002

23. De Craene B and Berx G: Regulatory networks defining EMT during cancer initiation and progression. Nat Rev Cancer 13: 97-110, 2013

24. Chaffer CL and Weinberg RA: A perspective on cancer cell metastasis. Science 331: 1559-1564, 2011

25. Hill B, De Melo J, Yan J, Kapoor A, He L, Cutz JC, Feng X, Bakhtyar $\mathrm{N}$ and Tang D: Common reduction of the Raf kinase inhibitory protein in clear cell renal cell carcinoma. Oncotarget 5: 7406-7419, 2014.
26. Langner C, Ratschek M, Rehak P, Schips L and Zigeuner R: Expression of MUC1 (EMA) and E-cadherin in renal cell carcinoma: a systematic immunohistochemical analysis of 188 cases. Mod Pathol 17: 180-188, 2004.

27. Clevers H: Wnt/beta-catenin signaling in development and disease. Cell 127: 469-480, 2006

28. Rubinfeld B, Albert I, Porfiri E, Fiol C, Munemitsu S and Polakis P: Binding of GSK3beta to the APC-beta-catenin complex and regulation of complex assembly. Science 272: 1023-1026, 1996.

29. Kimelman D and Xu W: beta-catenin destruction complex: Insights and questions from a structural perspective. Oncogene 25: 7482-7491, 2006

30. Taurin S, Sandbo N, Qin Y, Browning D and Dulin NO: Phosphorylation of beta-catenin by cyclic AMP-dependent protein kinase. J Biol Chem 281: 9971-9976, 2006.

31. Hino S, Tanji C, Nakayama KI and Kikuchi A: Phosphorylation of beta-catenin by cyclic AMP-dependent protein kinase stabilizes beta-catenin through inhibition of its ubiquitination. Mol Cell Biol 25: 9063-9072, 2005.

32. Coussens LM, Fingleton B and Matrisian LM: Matrix metalloproteinase inhibitors and cancer: Trials and tribulations. Science 295: 2387-2392, 2002

33. Sattler M and Salgia R: The MET axis as a therapeutic target. Update Cancer Ther 3: 109-118, 2009.

34. Grandori C, Cowley SM, James LP and Eisenman RN: The Myc/Max/Mad network and the transcriptional control of cell behavior. Annu Rev Cell Dev Biol 16: 653-699, 2000. 